\title{
On Factorization and Solutions of $q$-difference Equations Satisfied by some Classes of Orthogonal Polynomials
}

\author{
M. FOUPOUAGNIGNI ${ }^{a, \star}$, W. KOEPF ${ }^{b}$ and A. RONVEAUX \\ aUniversity of Yaounde I, Advanced School of Education, Department of Mathematics. \\ P.O. Box 47 Yaounde, Cameroon; ' University of Kassel, Department of Mathematics and \\ Computer Science, Heinrich-Plett Str. 40, 34132 Kassel, Germany; ${ }^{\circ}$ Facultés Universitaires \\ Notre Dame de la Paix, B-5000 Namur, Belgium
}

(Received 24 April 2003; Revised 16 October 2003; In final form 17 October 2003)

We derive and factorize the fourth-order $q$-difference equations satisfied by orthogonal polynomials obtained from some perturbations of the recurrence coefficients of $q$-classical orthogonal polynomials. These perturbations include the $r$ th associated, the anti-associated, the general co-recursive, co-recursive associated, co-dilated and the general co-modified $q$-classical orthogonal polynomials. Moreover we find a basis of four linearly independent solutions of these fourth-order $q$-difference equations and express the modified families in terms of the starting ones.

Keywords: q-Classical orthogonal polynomials; Functions of the second kind; Associated orthogonal polynomials; Perturbed orthogonal polynomials; Second and fourth-order $q$-difference equations

MSC 2000: 33D45; 33C45

\section{INTRODUCTION}

We consider polynomial families $\left(P_{\mathrm{n}}(x(s))\right)_{\mathrm{n}}$ of a discrete variable on a non-uniform lattice $x(s)=q^{s}$ or $x(s)=q^{-s}$ that are solutions of the second-order $q$-difference equation of hypergeometric type written in its equivalent form as $[25,26]$

$$
\sigma(x(s)) \frac{\Delta}{\Delta x\left(s-\frac{1}{2}\right)} \frac{\nabla}{\nabla x(s)} y(x(s))+\tau(x(s)) \frac{\Delta}{\Delta x(s)} y(x(s))+\lambda_{n} y(x(s))=0,
$$

where $\lambda_{n}$ is a constant. $\sigma(x(s)) \equiv \sigma(x)$ is a polynomial of degree at most 2 in the variable $x$ and $\tau(x)$ a first degree polynomial in $x . \Delta$ and $\nabla$ are the forward and the backward difference operators defined respectively by

$$
\Delta f(s)=f(s+1)-f(s), \quad \nabla f(s)=f(s)-f(s-1) .
$$

Under the boundary condition

$$
\left.\sigma(x(s)) w(x(s)) x^{k}\left(s-\frac{1}{2}\right)\right|_{s=a, b}=0, \quad \forall k,
$$

*Corresponding author. E-mail: foumama@yahoo.fr; foupoua@uycdc.uninet.cm 
the polynomials $\left(P_{\mathrm{n}}(x(s))\right)_{n}$ are orthogonal with respect to the weight function $w(x(s))$ $[25,28]$

$$
\sum_{s=a}^{b-1} P_{n}(x(s)) P_{m}(x(s)) w(x(s)) \Delta x\left(s-\frac{1}{2}\right)=k_{n} \delta_{n, m}, \quad k_{n} \neq 0, \forall n,
$$

where $\delta_{n, m}$ is the Kronecker symbol, and the weight function $w(x(s))$ defined on the interval $[a, b]$ satisfies the $q$-difference equation

$$
\frac{\Delta}{\Delta x\left(s-\frac{1}{2}\right)}(\sigma(x(s)) w(x(s)))=\tau(x(s)) w(x(s)) .
$$

Using the relations

$$
\left(\mathcal{D}-\mathcal{D}_{\frac{1}{q}}\right) f(x)=(q-1) x \mathcal{D}_{q} \mathcal{D}_{\frac{1}{q}} f(x), \quad \mathcal{D}_{\frac{1}{q}} \mathcal{D}_{q}=q \mathcal{D}_{q} \mathcal{D}_{\frac{1}{q}}
$$

where $\mathcal{D}_{q}$ is the Hahn operator [16] defined by

$$
\mathcal{D}_{q} f(x)=\frac{f(q x)-f(x)}{(q-1) x}, q \neq 1
$$

one transforms easily Eqs (1) and (2) respectively into (see for example, Ref. [23])

$$
\begin{gathered}
\mathbb{L}_{n}(y(x))=\phi(x) \mathcal{D}_{q} \mathcal{D}_{\frac{1}{q}} y(x)+\psi(x) \mathcal{D}_{q} y(x)+\lambda_{n} y(x)=0, \\
\mathcal{D}_{q}(\phi(x) w(x))=\psi(x) w(x) .
\end{gathered}
$$

The polynomials $\phi$ and $\psi$ are given by

$$
\phi(x)=q^{\frac{1}{2}} \sigma(x), \quad \psi(x)=\tau(x)
$$

for the lattice $x(s)=q^{s}$ and

$$
\phi(x)=q^{\frac{1}{2}} \sigma(x)-(q-1) x \tau(x), \quad \psi(x)=\tau(x)
$$

for the lattice $x(s)=q^{-s}$. The constant $\lambda_{n}$ reads

$$
\lambda_{n}=-[n]_{q}\left(\psi^{\prime}+[n-1]_{\frac{1}{2}} \frac{\phi^{\prime \prime}}{2 q}\right), \quad \psi^{\prime}(x)=\frac{\mathrm{d} \psi(x)}{\mathrm{d} x}, \quad[n]_{q}=\frac{q^{n}-1}{q-1} .
$$

Orthogonal polynomials $\left(P_{n}(x)\right)_{n}$ satisfying Eq. (4) or its equivalent form

$$
(\phi(x)+(q-1) x \psi(x)) \mathcal{D}_{q} \mathcal{D}_{\frac{1}{q}} y(x)+\psi(x) \mathcal{D}_{\frac{1}{q}} y(x)+\lambda_{n} y(x)=0
$$

are called $q$-classical [2,22,23]. In Ref. [23], $q$-classical orthogonal polynomials were identified among polynomial families belonging to the so-called $q$-Askey scheme [20]. They are: The Big $q$-Jacobi, Big $q$-Laguerre, Little $q$-Jacobi, Little $q$-Laguerre (Wall), $q$-Laguerre, Alternative $q$-Charlier, Al-Salam-Carlitz I, Al-Salam-Carlitz II, Stieltjes-Wigert, Discrete $q$-Hermite, Discrete $q^{-1}$-Hermite II, $q$-Hahn, $q$-Meixner, Quantum $q$-Krawtchouk, $q$-Krawtchouk, Affine $q$-Krawtchouk, and $q$-Charlier polynomials. 
Notice that the $q$-difference equation (4) can also be written in the following equivalent form which will be used in the next sections

$$
\begin{aligned}
\mathbb{D}_{n}(y(x))= & t(q x)^{2}\left(\mathbb{L}_{n}(y)\right)(q x) \\
= & (\phi(q x)+t(q x) \psi(q x)) y\left(q^{2} x\right)-((q+1) \phi(q x)+t(q x) \psi(q x) \\
& \left.-t(q x)^{2} \lambda_{n}\right) y(q x)+q \phi(q x) y(x)=0,
\end{aligned}
$$

with the notation $t(x)=(q-1) x$.

Polynomial solutions $\left(P_{n}(x)\right)_{n}$ of (4) (supposed monic: $P_{n}(x)=x^{n}+$ lower terms) satisfy a three-term recurrence equation

$$
P_{n+1}(x)=\left(x-\beta_{n}\right) P_{n}(x)-\gamma_{n} P_{n-1}(x), \quad n \geq 1,
$$

with the initial conditions

$$
P_{-1}(x)=0, \quad P_{0}(x)=1,
$$

where $\beta_{n}$ and $\gamma_{n}$ are real numbers with $\gamma_{n} \neq 0, \forall n \in \mathbb{N}$, and $\mathbb{N}$ denotes the set $\mathbb{N}=\{1,2, \ldots\}$. Also the notation $\mathbb{Z}_{+}=\{0,1,2, \ldots\}$ will be used.

When $\left(P_{n}\right)_{n}$ is $q$-classical, the coefficients $\beta_{n}$ and $\gamma_{n}$ are rational functions in $q^{n}$ and are given in terms of coefficients of the polynomials $\phi$ and $\psi$ appearing in Eq. (4) [23,19].

Some modifications of the three-term recurrence equation (8) lead to new families of orthogonal polynomials which satisfy a $q$-difference equation of order 4 instead of 2 . For example, the $r$ th associated $\left(P_{n}\right)_{n}$, denoted by $\left(P_{n}^{(r)}\right)_{n}$ and obtained by shifting the coefficients $\beta_{n}$ and $\gamma_{n}$ in Eq. (8)

$$
P_{n+1}^{(r)}(x)=\left(x-\beta_{n+r}\right) P_{n}^{(r)}(x)-\gamma_{n+r} P_{n-1}^{(r)}(x), \quad n \geq 1, \quad P_{-1}^{(r)}(x)=0, \quad P_{0}^{(r)}(x)=1,
$$

satisfy a fourth-order $q$-difference equation $[9,13]$ and cannot satisfy in general a secondorder $q$-difference equation $[9,13]$.

The fourth-order $q$-difference equation satisfied by some perturbations (listed in section "Preliminaries") of $q$-classical orthogonal polynomials, were given in Refs. [10,13] for the first and $r$ th associated, and in Refs. [14,15] for the co-recursive and the co-recursive associated $q$-classical orthogonal polynomials, respectively. Also, the factorization and the solution of the fourth-order differential and difference equations satisfied by some modifications of classical orthogonal polynomials of continuous and discrete variables were obtained recently in Refs. [11,12].

Following the above mentioned works [11,12], we derive in this paper the fourth-order $q$-difference equations satisfied by the above mentioned modifications of the three-term recurrence coefficients of the $q$-classical orthogonal polynomials. We also factorize the corresponding $q$-difference operators and find a basis of solutions for each case.

In the second section we define some types of perturbations we will deal with and recall important known results. The third section is devoted to the derivation and factorization of the corresponding fourth-order $q$-difference equations. In the fourth section we give a basis of four linear independent solutions of these $q$-difference equations and we also give some examples and applications.

The fifth section is devoted to the application of the results obtained to the $q$-Charlier and the Stieltjes-Wigert cases. We use the factorization obtained in the third section to solve 
a family of second-order $q$-difference equations and show how to extend Theorem 2 to real order of association.

\section{PRELIMINARIES}

We first present some examples of the perturbations we will deal with and recall known results we need.

Given $\left(P_{n}\right)_{n}$ a family of $q$-classical orthogonal polynomials, we deal with perturbations of the three-term recurrence equation which lead to a new family of orthogonal polynomials denoted by $\left(\bar{P}_{n}\right)_{n}$ related to the initial one by

$$
\bar{P}_{n}(x)=A_{n}(x) P_{n+k-1}^{(1)}(x)+B_{n}(x) P_{n+k}(x), \quad n \geq k^{\prime},
$$

where $A_{n}$ and $B_{n}$ are polynomials of degree uniformly bounded with respect to $n$, and $k$, $k^{\prime} \in \mathbb{Z}_{+} . P_{n}^{(1)}$ is the first associated of $P_{n}$ (see Eq. (9)).

Among these perturbations belong [21] (see also Refs. [11,12] for more details and intermediate relations):

1. The $r$ th associated orthogonal polynomials $\left(P_{n}^{(r)}\right)_{n}$ defined by the relation (9);

2. The generalized co-recursive orthogonal polynomials, denoted by $\left(P_{n}^{[k, \mu]}\right)_{n}$ and defined by

$$
\begin{aligned}
& P_{n+1}^{[k, \mu]}(x)=\left(x-\beta_{n}^{*}\right) P_{n}^{[k, \mu]}(x)-\gamma_{n} P_{n-1}^{[k, \mu]}(x), \quad n \geq 1, \\
& P_{0}^{[k, \mu]}(x)=1, \quad P_{1}^{[k, \mu]}(x)=x-\beta_{0}^{*},
\end{aligned}
$$

where $\beta_{n}^{*}=\beta_{n}$ for $n \neq k$ and $\beta_{k}^{*}=\beta_{k}+\mu$;

3. The generalized co-recursive associated, denoted by $\left(P_{n}^{\{r, k, \mu\}}\right)_{n}$, which is the generalized co-recursive (at the level $k$ ) of the $r$ th associated $\left(P_{n}^{(r)}\right)_{n}$;

4. The generalized co-dilated orthogonal polynomials, denoted by $\left(P_{n}^{|k, \lambda|}\right)_{n}$ and defined by the relation

$$
\begin{aligned}
& P_{n+1}^{|k, \lambda|}(x)=\left(x-\beta_{n}\right) P_{n}^{|k, \lambda|}(x)-\gamma_{n}^{*} P_{n-1}^{|k, \lambda|}(x), \quad n \geq 1, \\
& P_{0}^{|k, \lambda|}(x)=1, \quad P_{1}^{|k, \lambda|}(x)=x-\beta_{0},
\end{aligned}
$$

where $\gamma_{n}^{*}=\gamma_{n}$ for $n \neq k$ and $\gamma_{k}^{*}=\lambda \gamma_{k}$, with $\lambda \neq 0$.

5. The generalized co-modified orthogonal polynomials, denoted by $\left(P_{n}^{|k, \mu, \lambda|}\right)_{n}$ and defined by the three-term recurrence equation

$$
\begin{aligned}
& P_{n+1}^{[k, \mu, \lambda]}(x)=\left(x-\beta_{n}^{*}\right) P_{n}^{[k, \mu, \lambda]}(x)-\gamma_{n}^{*} P_{n-1}^{[k, \mu, \lambda]}(x), \quad n \geq 1, \\
& P_{0}^{[k, \mu, \lambda]}(x)=1, \quad P_{1}^{[k, \mu, \lambda]}(x)=x-\beta_{0}^{*},
\end{aligned}
$$

where $\beta_{n}^{*}=\beta_{n}, \gamma_{n}^{*}=\gamma_{n}$ for $n \neq k$ and $\beta_{k}^{*}=\beta_{k}+\mu, \gamma_{k}^{*}=\lambda \gamma_{k}$, with $\lambda \neq 0$.

6. The anti-associated orthogonal polynomials (see Ref. [27] for more details) denoted by $\left(P_{n}^{(-r)}\right)_{n}, r \in \mathbb{N}$ obtained by pushing down a given Jacobi matrix and by introducing in the empty upper left corner new coefficients $\beta_{-i}(i=r, r-1, r-2, \ldots, 0)$ on the diagonal and new coefficients $\gamma_{-i}(i=r-1, r-1, r-2, \ldots, 0)$, with $\gamma_{-i} \neq 0$ on the lower 
subdiagonal. This family can also be defined by the three-term recurrence equations

$$
\begin{aligned}
& P_{n+1}^{(-r)}(x)=\left(x-\beta_{-r+n}\right) P_{n}^{(-r)}(x)-\gamma_{-r+n} P_{n-1}^{(-r)}(x), \quad n \leq r-1, \\
& P_{-1}^{(-r)}(x)=0, \quad P_{0}^{(-r)}(x)=1,
\end{aligned}
$$

and

$$
P_{n+1}^{(-r)}(x)=\left(x-\beta_{n-r}\right) P_{n}^{(-r)}(x)-\gamma_{n-r} P_{n-1}^{(-r)}(x), \quad n>r
$$

The six examples of modifications listed above satisfy relation (10) and the coefficients $A_{n}(x)$ and $B_{n}(x)$ of Eq. (10) for each case are given in Refs. [11,12,21] for the first five cases and in Ref. [27] for the anti-associated.

Next we state the following results due to Suslov [28] (see also Refs. [3,17]). We also give the relation due to Foupouagnigni et al. giving the link between $P_{n}$ and its first associated.

\section{Known Results}

1. The two linearly independent solutions of the difference equation (1) on the lattice $x(s)=q^{s}$ or $x(s)=q^{-s}$ are the polynomials $P_{n}(x(s))$ orthogonal with respect to the weight function $w(x(s))$ (see Eq. (2)) and the function of the second kind $Q_{n}$ defined by Ref. [28]

$$
Q_{n}(x(z))=\frac{1}{w(x(z))} \sum_{s=a}^{b-1} \frac{P_{n}(x(s)) w(x(s)) \Delta x\left(s-\frac{1}{2}\right)}{x(s)-x(z)}, \quad z \notin\{a, a+1, \ldots b-1\} .
$$

2. $Q_{n}$ is related to $P_{n}$ and its first associated (associated of order $r=1$ ) by Ref. [28]

$$
Q_{n}(x(z))=P_{n}(x(z)) Q_{0}(x(z))+\frac{P_{n-1}^{(1)}(x(z))}{w(x(z))},
$$

and obeys the following asymptotic relation

$$
Q_{n}(x(z))=-\frac{\prod_{j=0}^{n} \gamma_{j}}{w(x(z)) x^{n+1}(z)}\left(1+O\left(\frac{1}{x(z)}\right)\right), \quad x(z) \rightarrow \infty,
$$

with

$$
\gamma_{0}=\sum_{s=a}^{b-1} w(x(s)) \Delta x\left(s-\frac{1}{2}\right) .
$$

3. $Q_{n}(x)$ satisfies the three-term recurrence equation (8) with the initial conditions [28]

$$
Q_{-1}(x(z))=\frac{-1}{w(x(z))}, \quad Q_{0}(x(z))=Q_{0}\left(x\left(z_{0}\right)\right)+C \sum_{s=z_{0}+1}^{z} \frac{\nabla x(s)}{\sigma(x(s)) w(x(s))},
$$

where $C$ is a constant.

4. Foupouagnigni et al. [10] proved that for all families of $q$-classical orthogonal polynomials $\left(P_{n}(x)\right)_{n}$ satisfying Eq. (4), $P_{n}$ and its first associated $P_{n}^{(1)}$ are linked by 
the relation

$$
\mathbb{D}_{n}^{*}\left(P_{n-1}^{(1)}(x)\right)=a_{1}(x) P_{n}(q x)+a_{0}(x) P_{n}(x),
$$

where $\mathbb{D}_{n}^{*}$ is the $q$-difference operator given by

$$
\begin{aligned}
& \mathbb{D}_{n}^{*}(y(x))=(\phi(q x)+t(q x) \psi(q x))\left(\phi\left(q^{2} x\right) y\left(q^{2} x\right)\right. \\
& -\left((1+q) \phi(q x)+t(q x) \psi(q x)-t(q x)^{2} \lambda_{n}\right) y(q x)+q(\phi(x) \\
& \quad+t(x) \psi(x)) y(x))
\end{aligned}
$$

with the polynomials $a_{i}, i=0,1$ being

$$
\begin{aligned}
& a_{1}(x)=\left(\frac{\phi^{\prime \prime}}{2}-\psi^{\prime}\right)\left((1+q) \phi(q x)+t(q x) \psi(q x)-t(q x)^{2} \lambda_{n}\right) t(q x), \\
& a_{0}(x)=-\left(\frac{\phi^{\prime \prime}}{2}-\psi^{\prime}\right)((1+q) \phi(q x)+t(q x) \psi(q x)) t(q x) .
\end{aligned}
$$

Notice also the important relation

$$
\phi(q x) \mathbb{D}_{n}^{*}(w(x) y(x))=w(x)(\phi(x)+t(x) \psi(x))(\phi(q x)+t(q x) \psi(q x)) \mathbb{D}(y(x)), \quad \forall y,
$$

where $w$ is the weight function satisfying Eq. (5) and $\mathbb{D}_{n}$ the $q$-difference operator given by Eq. (7).

\section{DERIVATION OF THE $q$-DIFFERENCE EQUATIONS AND FACTORIZATION}

Given $\left(P_{n}\right)_{n}$ a $q$-classical orthogonal polynomial sequence, we consider in general all transformations which lead to new families of orthogonal polynomials denoted by $\left(\bar{P}_{n}\right)_{n}$ and are related to the starting sequence by Eq. (10). We have the following:

THEOREM 1 1- The orthogonal polynomials $\left(\bar{P}_{n}\right)_{n \geq k^{\prime}}$ satisfy a common fourth-order linear q-difference equation

$$
\mathbb{F}_{n}(y(x))=\left(J_{4}(x, n) \mathcal{G}_{q}^{4}+J_{3}(x, n) \mathcal{G}_{q}^{3}+J_{2}(x, n) \mathcal{G}_{q}^{2}+J_{1}(x, n) \mathcal{G}_{q}+J_{0}(x, n)\right) y(x)=0,
$$

where the coefficients $J_{i}$ are polynomials in $x$, with degree not depending on $n$ and $\mathcal{G}_{q}$ the $q$-shift operator defined by

$$
\mathcal{G}_{q} f(x)=f(q x)
$$

2- The operator $\mathbb{F}_{n}$ can be factored as product of two second-order linear q-difference operators $\mathbb{S}_{n}$ and $\mathbb{T}_{n}$ :

$$
X_{n} \mathbb{F}_{n}=\mathbb{S}_{n} \mathbb{\mathbb { T }}_{n}, \quad n \geq k,
$$

where $X_{n}$ and the coefficients in $\mathbb{S}_{n}$ and $\mathbb{T}_{n}$ are polynomials in $x$ of degree not depending on $n$.

Proof In the first step, we solve Eq. (10) in terms of $P_{n+k-1}^{(1)}$

$$
P_{n+k-1}^{(1)}(x)=\frac{\bar{P}_{n}(x)-B_{n}(x) P_{n+k(x)}}{A_{n}(x)},
$$


and substitute the previous relation in Eq. (16) in which $n$ is replaced by $n+k$. Then we use Eq. (7) (for $P_{n+k}$ ) to eliminate the term $P_{n+k}\left(q^{2} x\right)$ and get

$$
\mathbb{M}_{n+k}\left(\bar{P}_{n}\right)=b_{1} P_{n+k}(q x)+b_{0} P_{n+k}(x),
$$

where $b_{i}$ are rational functions of $x$ and $\mathbb{M}_{n+k}$ a second-order linear $q$-difference operator given in terms of operator $\mathbb{D}_{n+k}^{*}$ (see Eq. (16)) by

$$
\mathbb{M}_{n+k}(y)=A_{n}(x) A_{n}(q x) A_{n}\left(q^{2} x\right) \mathbb{D}_{n+k}^{*}\left(\frac{y}{A_{n}}\right) .
$$

Next, we shift Eq. (19) and again use Eq. (7), to eliminate $P_{n+k}\left(q^{2} x\right)$, and get

$$
\mathcal{G}_{q} \mathbb{M}_{n+k}\left(\bar{P}_{n}\right)=c_{1} P_{n+k}(q x)+c_{0} P_{n+k}(x) .
$$

We reiterate the same process using the previous equation and get

$$
\mathcal{G}_{q}^{2} \mathbb{M}_{n+k}\left(\bar{P}_{n}\right)=d_{1} P_{n+k}(q x)+d_{0} P_{n+k}(x),
$$

where $c_{i}$ and $d_{i}$ are again rational functions of $x$.

From Eqs. (19), (21) and (22), the following fourth-order $q$-difference equation holds, in a similar way as in Ref. [4]:

$$
\mathbb{F}_{n, 0}\left(\bar{P}_{n}\right)=\left|\begin{array}{ccc}
b_{1} & b_{0} & \mathbb{M}_{n+k}\left(\bar{P}_{n}\right) \\
c_{1} & c_{0} & \mathcal{G}_{q} \mathbb{M}_{n+k}\left(\bar{P}_{n}\right) \\
d_{1} & d_{0} & \mathcal{G}_{q}^{2} \mathbb{M}_{n+k}\left(\bar{P}_{n}\right)
\end{array}\right|=0 .
$$

After cancellation of the denominators, the previous equation can be written as

$$
\mathbb{F}_{n, 0}\left(\bar{P}_{n}\right)=e_{2} \mathcal{G}_{q}^{2} \mathbb{M}_{n+k}\left(\bar{P}_{n}\right)+e_{1} \mathcal{G}_{q} \mathbb{M}_{n+k}\left(\bar{P}_{n}\right)+e_{0} \mathbb{M}_{n+k}\left(\bar{P}_{n}\right)=\left[\mathbb{S}_{n, 0} \mathbb{\mathbb { T }}_{n}\right]\left(\bar{P}_{n}\right)=0,
$$

where the second-order $q$-difference operators $\mathbb{S}_{n, 0}$ and $\mathbb{T}_{n}$ are given by

$$
\mathbb{S}_{n, 0}=e_{2} \mathcal{G}_{q}^{2}+e_{1} \mathcal{G}_{q}+e_{0} \rrbracket, \quad \mathbb{T}_{n}=\mathbb{M}_{n+k},
$$

with $e_{i}, i=0,1,2$ being polynomials in $x$. We conclude the proof by noticing that after cancelling common factors of the polynomial coefficients of the $q$-difference operators $\mathbb{F}_{n, 0}$ and $\mathbb{S}_{n, 0}$ appearing in Eq. (23), we obtain the factorization

$$
X_{n} \mathbb{F}_{n}=\mathbb{S}_{n} \mathbb{T}_{n},
$$

where $X_{n}$ is a polynomial of degree not depending on $n, \mathbb{F}_{n}$ and $\mathbb{S}_{n}$ are the $q$-difference operators of order 4 and 2 (with polynomial coefficients) obtained respectively from $\mathbb{F}_{n, 0}$ and $\mathbb{S}_{n, 0}$ by cancelling common factors and taking care that the polynomial $X_{n}$ and the polynomial coefficients of $\mathbb{F}_{n}$ as well as those of $\mathbb{S}_{n}$ have no common factor.

We would like to mention that the factorization of the fourth-order $q$-difference equation satisfied by the first associated (i.e. $r=1$ ) $q$-classical orthogonal polynomials was derived in Ref. [10]. The previous theorem factorizing the fourth-order $q$-difference equation satisfied by orthogonal polynomials $\left(\bar{P}_{n}\right)_{n}$ verifying Eq. (10) is a new result. For example, it can be used to deduce the factorized form of the fourth-order $q$-difference equation satisfied by the $r$ th associated $q$-classical orthogonal polynomials for any positive integer $r$. An application of this result will be given in section "Specializations and Application". 
In what follows, we will denote respectively by $\mathbb{F}_{n}^{(r)}, \mathbb{F}_{n}^{[k, \mu]}, \mathbb{F}_{n}^{\{r, k, \mu\}} \mathbb{F}_{n}^{|k, \lambda|}$ and $\mathbb{F}_{n}^{[k, \mu, \lambda]}$ the fourth-order $q$-difference operators for the $r$ th associated, the generalized co-recursive, the generalized co-recursive associated, the generalized co-dilated, and the generalized co-modified orthogonal polynomials. $\mathbb{S}_{n}^{(r)}, \mathbb{T}_{n}^{(r)}$ and $X_{n}^{(r)}$ represent respectively the secondorder $q$-difference operators and the polynomial factor appearing in Eq. (25) for the $r$ th associated case.

COROLLARY 1 The fourth-order q-difference operator $\mathbb{F}_{n}^{(r)}$ for the rth associated q-classical orthogonal polynomials factorizes as

$$
\mathbb{S}_{n}^{(r)} \mathbb{\mathbb { T }}_{n}^{(r)}=X_{n}\left(\sigma, \tau, P_{r-1}, \lambda_{r-1}\right) \mathbb{F}_{n}^{(r)},
$$

where $X_{n} \equiv X_{n}\left(\sigma, \tau, P_{r-1}, \lambda_{r-1}\right)$ is a polynomial depending on $\sigma, \tau, P_{r-1}$ and $\lambda_{r-1}$.

The fourth-order q-difference operator $\mathbb{F}_{n}^{(r)}$ can also be factorized as

$$
\tilde{\mathbb{S}}_{n}^{(r)} \tilde{\mathbb{T}}_{n}^{(r)}=\tilde{X}_{n}\left(\sigma, \tau, Q_{r-1}, \lambda_{r-1}\right) \mathbb{F}_{n}^{(r)},
$$

where the expression $\tilde{X}_{n}\left(\sigma, \tau, Q_{r-1}, \lambda_{r-1}\right)$ and the operators $\tilde{\mathbb{S}}_{n}^{(r)}$ and $\tilde{\mathbb{T}}_{n}^{(r)}$ are obtained from the expression $X_{n}\left(\sigma, \tau, P_{r-1}, \lambda_{r-1}\right)$ and operators $\mathbb{S}_{n}^{(r)}$ and $\mathbb{T}_{n}^{(r)}$ (see Eq. (26)) respectively by replacing the polynomials $P_{r-1}$ with the function $Q_{r-1}$.

For all $q$-classical orthogonal polynomial families, we have computed using the software Maple 8 [24] the expression of the polynomial $X\left(\sigma, \tau, P_{r-1}, \lambda_{r-1}\right)$ and also the coefficients of the operators $\mathbb{F}_{n}^{(r)}, \mathbb{S}_{n}^{(r)}$ and $\mathbb{T}_{n}^{(r)}$. Since these results are too large and space consuming we will give them only for the $q$-Charlier and for the Stieltjes-Wigert cases (in section "Specializations and Application").

\section{SOLUTIONS OF THE FOURTH-ORDER $q$-DIFFERENCE EQUATIONS}

In the following, we solve the fourth-order $q$-difference equation satisfied by the five perturbations listed in the section "Preliminaries" and represent the new families of orthogonal polynomials in terms of solutions of second-order $q$-difference equations. It shall be noticed that the results given in the following theorem (Theorem 2) were recently obtained by Ismail and Simeonov using a different approach [18].

THEOREM 2 Let $\left(P_{n}\right)_{n}$ be a q-classical orthogonal polynomial sequence, $r \in \mathbb{Z}_{+}$and $\left(P_{n}^{(r)}\right)_{n}$ the rth associated of $\left(P_{n}\right)_{n}$. Four linearly independent solutions of the difference equation

$$
\mathbb{F}_{n}^{(r)}(y)=0,
$$

satisfied by $\left(P_{n}^{(r)}\right)_{n}$ are

$$
\begin{aligned}
& A_{n}^{(r)}(x)=w(x) P_{r-1}(x) P_{n+r}(x), \\
& B_{n}^{(r)}(x)=w(x) P_{r-1}(x) Q_{n+r}(x), \\
& C_{n}^{(r)}(x)=w(x) Q_{r-1}(x) P_{n+r}(x), \\
& D_{n}^{(r)}(x)=w(x) Q_{r-1}(x) Q_{n+r}(x),
\end{aligned}
$$


$Q_{n}$ denoting the function of second kind associated to $\left(P_{n}\right)_{n}$ which is defined by Eq. (12). Moreover, $P_{n}^{(r)}$ is related to these solutions by

$$
\begin{aligned}
P_{n}^{(r)}(x) & =\frac{B_{n}^{(r)}(x)-C_{n}^{(r)}(x)}{\gamma_{0} \Gamma_{r-1}} \\
& =\frac{w(x)\left(P_{r-1}(x) Q_{n+r}(x)-Q_{r-1}(x) P_{n+r}(x)\right)}{\gamma_{0} \Gamma_{r-1}}, \quad \forall n \in \mathbb{N}, \quad \forall r \in \mathbb{Z}_{+},
\end{aligned}
$$

where $\gamma_{0}$ given by Eq. (14) and $\Gamma_{k}$ is given by

$$
\Gamma_{k}=\prod_{i=1}^{n} \gamma_{i}, \quad \Gamma_{0} \equiv 1 .
$$

Proof In the first step, we solve the $q$-difference equation

$$
\mathbb{T}_{n}^{(r)}(y)=0 .
$$

To do this, we use Eqs. (18), (20) and (24) as well as the relation [7]

$$
P_{n}^{(r)}(x)=\frac{P_{r-1}(x)}{\Gamma_{r-1}} P_{n+r-1}^{(1)}(x)-\frac{P_{r-2}^{(1)}(x)}{\Gamma_{r-1}} P_{n+r}(x), \quad n \geq 0, \quad r \geq 2,
$$

to get

$$
\begin{aligned}
\mathbb{T}_{n}^{(r)}(y)= & \mathbb{M}_{n+r}(y)=P_{r-1}(x) P_{r-1}(q x) P_{r-1}\left(q^{2} x\right) \mathbb{D}_{n+r}^{*}\left(\frac{y}{P_{r-1}}\right) \\
= & P_{r-1}(x) P_{r-1}(q x) P_{r-1}\left(q^{2} x\right) w(x)(\phi(x)+t(x) \psi(x)) \\
& \times(\phi(q x)+t(q x) \psi(q x)) \mathbb{D}_{n+r}(z) / \phi(q x),
\end{aligned}
$$

where the functions $y$ and $z$ are related by $y(x)=z(x) w(x) P_{r-1}(x)$. Since the two linearly independent solutions of $\mathbb{D}_{n+r}(z)=0$ are $P_{n+r}$ and $Q_{n+r}$ (see section "Preliminaries"), the two linearly independent solutions of $\mathbb{T}_{n}^{(r)}(y)=0$, (which are also solutions of Eq. (28) thanks to the factorization $X \mathbb{F}_{n}^{(r)}=\mathbb{S}_{n}^{(r)} \mathbb{T}_{n}^{(r)}$ are

$$
\begin{aligned}
& A_{n}^{(r)}(x)=w(x) P_{r-1}(x) P_{n+r}(x), \\
& B_{n}^{(r)}(x)=w(x) P_{r-1}(x) Q_{n+r}(x) .
\end{aligned}
$$

Using Eqs. (27) and (30) and the fact that the function $Q_{n}$ and the weight function $w$ satisfy Eqs. (4) and (5) respectively, leads to

$$
\begin{gathered}
\tilde{\mathbb{T}}_{n}^{(r)}(y)=Q_{r-1}(x) Q_{r-1}(q x) Q_{r-1}\left(q^{2} x\right) w(x)(\phi(x)+t(x) \psi(x)) \\
\times(\phi(q x)+t(q x) \psi(q x)) \mathbb{D}_{n+r}(z) / \phi(q x),
\end{gathered}
$$

where the functions $y$ and $z$ are related by $y(x)=z(x) w(x) Q_{r-1}(x)$. Equation (27) permits us to conclude that the two independent solutions of $\tilde{\mathbb{T}}_{n}^{(r)}(y)=0$ (which are also solutions of 
Eq. (28) due to Eq. (26)) are given by

$$
\begin{aligned}
& C_{n}^{(r)}(x)=w(x) Q_{r-1}(x) P_{n+r}(x), \\
& D_{n}^{(r)}(x)=w(x) Q_{r-1}(x) Q_{n+r}(x) .
\end{aligned}
$$

The four obtained solutions of Eq. (28) are linearly independent since $P_{n}$ and $Q_{n}$ are two linearly independent solutions of Eq. (4) and have different asymptotic behavior (see Eq. (14)).

Next, we state the following theorems for the generalized co-recursive, co-recursive associated, co-dilated and co-modified $q$-classical orthogonal polynomials. The proofs are similar to the previous ones and are also similar to those given in Refs. [11,12] for the continuous and discrete cases. However, one will need the following relations [11,12] linking the modified families to the starting ones.

$$
\begin{aligned}
P_{n}^{[k, \mu]}(x)= & -\frac{\mu P_{k}^{2}(x)}{\Gamma_{k}} P_{n-1}^{(1)}(x)+\left(1+\frac{\mu P_{k}(x) P_{k-1}^{(1)}}{\Gamma_{k}}\right) P_{n}(x), n \geq k+1 \\
P_{n}^{\{r, k, \mu\}}(x)= & \left(\frac{P_{r-1}(x)}{\Gamma_{r-1}}-\frac{\mu P_{k+r}(x) P_{k}^{(r)}(x)}{\Gamma_{r+k}}\right) P_{n+r-1}^{(1)}(x) \\
& -\left(\frac{P_{r-2}^{(1)}(x)}{\Gamma_{r-1}}-\frac{\mu P_{k+r-1}^{(1)}(x) P_{k}^{(r)}(x)}{\Gamma_{r+k}}\right) P_{n+r}(x), \quad n \geq k+1 ; \\
P_{n}^{[k, \lambda \mid}(x)= & \left(1-\frac{(1-\lambda) P_{k-1}(x) P_{k-1}^{(1)}}{\Gamma_{k-1}}\right) P_{n}(x) \\
& +\frac{(1-\lambda) P_{k-1}(x) P_{k}(x)}{\Gamma_{k-1}} P_{n-1}^{(1)}(x), \quad n \geq k+1, \\
& +\left(\frac{(1-\lambda) P_{k-1}(x) P_{k}(x)}{\Gamma_{k-1}}-\frac{\mu P_{k}^{2}(x)}{\Gamma_{k}}\right) P_{n-1}^{(1)}(x), \quad n \geq k+1 .
\end{aligned}
$$

THEOREM 3 Let $\left(P_{n}\right)_{n}$ be a q-classical orthogonal polynomial sequence, $k \in \mathbb{N}$ and $\left(P_{n}^{[k, \mu]}\right)_{n}$ the generalized co-recursive of $\left(P_{n}\right)_{n}$. Four linearly independent solutions of the q-difference equation

$$
\mathbb{F}_{n}^{[k, \mu]}(y)=0, \quad n \geq k+1,
$$

satisfied by $\left(P_{n}^{[k, \mu]}\right)_{n}$, are (with $n \geq k+1$ )

$$
\begin{aligned}
A_{n}^{[k, \mu]}(x) & =w(x) P_{k}^{2}(x) P_{n}(x), \\
B_{n}^{[k, \mu]}(x) & =w(x) P_{k}^{2}(x) Q_{n}(x), \\
C_{n}^{[k, \mu]}(x) & =\left[\gamma_{0} \Gamma_{k}+\mu w(x) P_{k}(x) Q_{k}(x)\right] P_{n}(x), \\
D_{n}^{[k, \mu]}(x) & =\left[\gamma_{0} \Gamma_{k}+\mu w(x) P_{k}(x) Q_{k}(x)\right] Q_{n}(x),
\end{aligned}
$$

where $Q_{n}$ is the function of second kind associated to $\left(P_{n}\right)_{n}$ defined by Eq. (12). 
Moreover, $P_{n}^{[k, \mu]}$ is related to these solutions by

$$
P_{n}^{[k, \mu]}=\frac{\left[\gamma_{0} \Gamma_{k}+\mu w(x) P_{k}(x) Q_{k}(x)\right] P_{n}(x)-\mu w(x) P_{k}^{2}(x) Q_{n}(x)}{\gamma_{0} \Gamma_{k}}, \quad k \geq 0, \quad n \geq k+1 .
$$

THEOREM 4 Let $\left(P_{n}\right)_{n}$ be a q-classical orthogonal polynomial sequence, $k \in \mathbb{N}$ and $\left(P_{n}^{|k, \lambda|}\right)_{n}$ the generalized co-dilated of $\left(P_{n}\right)_{n}$. Four linearly independent solutions of the difference equation

$$
\mathbb{F}_{n}^{|k, \lambda|}(y)=0, \quad n \geq k+1,
$$

satisfied by $\left(P_{n}^{|k, \lambda|}\right)_{n}$ are (with $n \geq k+1$ )

$$
\begin{aligned}
& A_{n}^{|k, \lambda|}(x)=w(x) P_{k-1}(x) P_{k}(x) P_{n}(x), \\
& B_{n}^{|k, \lambda|}(x)=w(x) P_{k-1}(x) P_{k}(x) Q_{n}(x), \\
& C_{n}^{|k, \lambda|}(x)=\left[\gamma_{0} \Gamma_{k}+(\lambda-1) \gamma_{k} w(x) P_{k-1}(x) Q_{k}(x)\right] P_{n}(x), \\
& D_{n}^{|k, \lambda|}(x)=\left[\gamma_{0} \Gamma_{k}+(\lambda-1) \gamma_{k} w(x) P_{k-1}(x) Q_{k}(x)\right] Q_{n}(x) .
\end{aligned}
$$

The co-dilated $P_{n}^{|k, \lambda|}$ is related to these solutions by

$$
\begin{aligned}
P_{n}^{|k, \lambda|}= & \frac{\left[\gamma_{0} \Gamma_{k}+(\lambda-1) \gamma_{k} w(x) P_{k-1}(x) Q_{k}(x)\right] P_{n}(x)-(\lambda-1) \gamma_{k} w(x) P_{k-1}(x) P_{k}(x) Q_{n}(x)}{\gamma_{0} \Gamma_{k}}, \\
& n \geq k+1
\end{aligned}
$$

THEOREM 5 Let $\left(P_{n}\right)_{n}$ be a q-classical orthogonal polynomial sequence, $k \in \mathbb{N}, r \in \mathbb{Z}_{+}$ and $\left(P_{n}^{\{r, k, \mu\}}\right)_{n}$ the generalized co-recursive associated of $\left(P_{n}\right)_{n}$. Four linearly independent solutions of the q-difference equation

$$
\mathbb{F}_{n}^{\{r, k, \mu\}}(y)=0, \quad n \geq k+1,
$$

satisfied by $\left(P_{n}^{\{r, k, \mu\}}\right)_{n}$ are (with $\left.n \geq k+1\right)$

$$
\begin{aligned}
& A_{n}^{\{r, k, \mu\}}(x)=\left(\gamma_{0} \Gamma_{k+r} P_{r-1}(x)-\mu w(x) P_{k+r}(x)\right. \\
& {\left.\left[P_{r-1}(x) Q_{k+r}(x)-Q_{r-1}(x) P_{k+r}(x)\right]\right) w(x) P_{n+r}(x), } \\
& B_{n}^{\{r, k, \mu\}}(x)=\left(\gamma_{0} \Gamma_{k+r} P_{r-1}(x)-\mu w(x) P_{k+r}(x)\right. {\left.\left[P_{r-1}(x) Q_{k+r}(x)-Q_{r-1}(x) P_{k+r}(x)\right]\right) w(x) Q_{n+r}(x), } \\
& C_{n}^{\{r, k, \mu\}}(x)=\left(\gamma_{0} \Gamma_{k+r} Q_{r-1}(x)-\mu w(x) Q_{k+r}(x)\right. {\left.\left[P_{r-1}(x) Q_{k+r}(x)-Q_{r-1}(x) P_{k+r}(x)\right]\right) w(x) P_{n+r}(x), } \\
& D_{n}^{\{r, k, \mu\}}(x)=\left(\gamma_{0} \Gamma_{k+r} Q_{r-1}(x)-\mu w(x) Q_{k+r}(x)\right. \\
& {\left.\left[P_{r-1}(x) Q_{k+r}(x)-Q_{r-1}(x) P_{k+r}(x)\right]\right) w(x) Q_{n+r}(x) . }
\end{aligned}
$$


Moreover, $P_{n}^{\{r, k, \mu\}}$ is related to these solutions by

$$
\begin{aligned}
P_{n}^{\{r, k, \mu\}}= & \left(\frac{P_{r-1}(x)}{\gamma_{0} \Gamma_{r-1}}-\frac{\mu w(x) P_{k+r}(x)\left[P_{r-1}(x) Q_{k+r}(x)-Q_{r-1}(x) P_{k+r}(x)\right]}{\gamma_{0}^{2} \Gamma_{r-1} \Gamma_{k+r}}\right) w(x) Q_{n+r}(x) \\
& -\left(\frac{Q_{r-1}(x)}{\gamma_{0} \Gamma_{r-1}}-\frac{\mu w(x) Q_{k+r}(x)\left[P_{r-1}(x) Q_{k+r}(x)-Q_{r-1}(x) P_{k+r}(x)\right]}{\gamma_{0}^{2} \Gamma_{r-1} \Gamma_{k+r}}\right) w(x) P_{n+r}(x), \\
& \quad r \geq 1, \quad n \geq k+1 .
\end{aligned}
$$

THEOREM 6 Let $\left(P_{n}\right)_{n}$ be a q-classical orthogonal polynomial sequence, $k \in \mathbb{N}$ and $\left(P_{n}^{[k, \mu, \lambda]}\right)_{n}$ the generalized co-modified of $\left(P_{n}\right)_{n}$. Four linearly independent solutions of the $q$-difference equation

$$
\mathbb{F}_{n}^{[k, \mu, \lambda]}(y)=0, \quad n \geq k+1
$$

satisfied by $\left(P_{n}^{[k, \mu, \lambda]}\right)_{n}$ are (with $\left.n \geq k+1\right)$

$$
\begin{aligned}
& A_{n}^{[k, \mu, \lambda]}(x)=\left[(\lambda-1) \gamma_{k} P_{k-1}(x) P_{k}(x)+\mu P_{k}^{2}(x)\right] w(x) P_{n}(x), \\
& B_{n}^{[k, \mu, \lambda]}(x)=\left[(\lambda-1) \gamma_{k} P_{k-1}(x) P_{k}(x)+\mu P_{k}^{2}(x)\right] w(x) Q_{n}(x), \\
& C_{n}^{[k, \mu, \lambda]}(x)=\left[\gamma_{0} \Gamma_{k}+(\lambda-1) \gamma_{k} w(x) P_{k-1}(x) Q_{k}(x)+\mu w(x) P_{k}(x) Q_{k}(x)\right] P_{n}(x), \\
& D_{n}^{[k, \mu, \lambda]}(x)=\left[\gamma_{0} \Gamma_{k}+(\lambda-1) \gamma_{k} w(x) P_{k-1}(x) Q_{k}(x)+\mu w(x) P_{k}(x) Q_{k}(x)\right] Q_{n}(x)
\end{aligned}
$$

The co-dilated $P_{n}^{[k, \mu, \lambda]}$ is related to these solutions by

$$
\begin{aligned}
P_{n}^{[k, \mu, \lambda]}= & \left(1+\frac{(\lambda-1) \gamma_{k} w(x) P_{k-1}(x) Q_{k}(x)+\mu w(x) P_{k}(x) Q_{k}(x)}{\gamma_{0} \Gamma_{k}}\right) P_{n}(x) \\
& -\frac{(\lambda-1) \gamma_{k} w(x) P_{k-1}(x) P_{k}(x)+\mu w(x) P_{k}^{2}(x)}{\gamma_{0} \Gamma_{k}} Q_{n}(x), \quad n \geq k+1 .
\end{aligned}
$$

\section{SPECIALIZATIONS AND APPLICATION}

In this section, doing computations with Maple 8 [24], we give explicitly the fourth-order $q$-difference equation (and its factorized form) satisfied by the $q$-Charlier and the StieltjesWigert polynomials. We also give data as well as second-order $q$-difference equation and the three-term recurrence equation for the $q$-Charlier and the Stieltjes-Wigert orthogonal polynomials [20]. Notice that the data on the $q$-Charlier polynomials defined on the lattice $x(s)=q^{s}$ are given in Ref. [1]. 


\section{The $q$-Charlier Polynomials}

The $q$-Charlier polynomials are defined by

$$
C_{n}\left(q^{-s} ; a ; q\right)=\left(-a^{-1} q ; q\right)_{n 1} \Phi_{1}\left(\begin{array}{c}
q^{-n} \\
-a^{-1} q
\end{array} \mid q ;-a^{-1} q^{n+1-s}\right),
$$

where

$$
(a ; q)_{n}=(1-a)(1-q a) \ldots\left(1-q^{n-1} a\right)
$$

and

$$
{ }_{1} \Phi_{1}\left(\begin{array}{l}
a \\
b
\end{array} \mid q ; x\right)=\sum_{k=0}^{\infty} \frac{(a ; q)_{k}}{(b ; q)_{k}}(-1)^{k} q^{\frac{k(k-1)}{2}} \frac{x^{k}}{(q ; q)_{k}} .
$$

The monic $q$-Charlier polynomials, denoted by $\left(P_{n}\right)_{n}$, are related to the $q$-Charlier polynomials by

$$
C_{n}\left(q^{-s} ; a ; q\right)=(-a)^{-n} q^{n^{2}} P_{n}(x), \quad x=q^{-s},
$$

and satisfy the following three-term recurrence equation

$$
\begin{aligned}
x P_{n}(x)= & P_{n+1}(x)+\left[1+q^{-1-2 n}\left\{a+q\left(1-q^{n}\right)\left(a+q^{n}\right)\right\}\right] \\
& \times P_{n}(x)+a q^{1-4 n}\left(1-q^{n}\right)\left(a+q^{n}\right) P_{n-1}(x) .
\end{aligned}
$$

$P_{n}$ satisfies the second-order $q$-difference equation

$$
(q x-1) y\left(q^{2} x\right)-\left(q^{n+1} x+a-1\right) y(q x)+a y(x)=0,
$$

which is equivalent to Eq. (4) with

$$
\phi(x)=a x, \quad \psi(x)=\frac{(x-1) q-a}{q-1}, \quad \lambda_{n}=\frac{\left(1-q^{n}\right) q}{(1-q)^{2}} .
$$

The $q$-Charlier weight $w(x)$ given by

$$
w(x)=\frac{a^{s} q^{\frac{s(s+1)}{2}}}{(q ; q)_{s}}, \quad x=q^{-s}
$$

satisfies the $q$-Pearson equation (5) with the polynomials $\phi$ and $\psi$ given as in Eq. (33).

The fourth-order $q$-difference operator $\mathbb{F}_{n}^{(r)}$ for the $r$ th associated $q$-Charlier orthogonal polynomials is given by

$$
\begin{aligned}
\mathbb{F}_{n}^{(r)}= & I_{4}(r, n, q, x) \mathcal{G}_{q}^{4}+I_{3}(r, n, q, x) \mathcal{G}_{q}^{3}+I_{2}(r, n, q, x) \mathcal{G}_{q}^{2} \\
& +I_{1}(r, n, q, x) \mathcal{G}_{q}+I_{0}(r, n, q, x)
\end{aligned}
$$


with the notations: $\nu=q^{r}, \rho=q^{n}$ and

$$
\begin{aligned}
I_{0}(r, n, q, x)= & (x-1) a q\left(-1+a-q+a q+\nu x q^{2}+q^{3} x \rho \nu\right) \\
I_{1}(r, n, q, x)= & -q^{4} \nu^{3} \rho(q \rho+1) x^{3}-q^{2} \nu^{2}\left(q \rho+2 q^{2} \rho+q^{3} \rho^{2}+q+\rho\right)(-1+a) x^{2} \\
& -q(-1+a)\left(q \nu a-a q^{2}+q^{2} \rho \nu a+a q^{2} \nu+q \rho \nu a+a+q^{3} \rho \nu a+\nu a-q \nu\right. \\
& \left.-q \rho \nu-\nu-q^{2} \rho \nu-\nu q^{2}-q^{3} \rho \nu\right) x-(1+q)(-1+a)\left(a^{2} q-a-a q+q\right) ; \\
I_{2}(r, n, q, x)= & q^{4} \nu^{3}(q \rho+1)\left(q^{2} \rho^{2}+1\right) x^{3}+(1+q) \nu q^{2}\left(-q^{3} \rho^{2} \nu+q^{3} \nu \rho^{2} a+q^{2} \rho \nu a\right. \\
& +q^{2} \nu \rho^{2} a-q^{2} \rho^{2} \nu-q^{2} \rho \nu-q^{2} \rho a+q \nu a+q \rho \nu a-q \rho \nu-q \nu-a q-\nu \\
& +\nu a) x^{2}+q\left(-2 a^{2} q^{2}+2 a^{2} q^{3} \rho \nu+2 a q^{2}+2 a q+2 q^{2} \rho \nu a^{2}-2 a^{2} q-3 \nu a\right. \\
& -4 q \nu a+2 a^{2} \nu+2 \nu q^{2}+2 a^{2} q \nu+2 a^{2} q^{2} \nu+2 \nu+2 q^{3} \rho \nu-4 q^{2} \rho \nu a \\
& \left.+2 q \nu+2 q^{2} \rho \nu-3 q \rho \nu a+2 q \rho \nu-3 a q^{2} \nu-3 q^{3} \rho \nu a+2 q \rho \nu a^{2}\right) x \\
& +(1+q)^{2}(-1+a)\left(a^{2}+1-a\right) ; \\
& -q^{6} \nu^{3} \rho(q \rho+1) x^{3}-q^{3} \nu^{2}\left(q^{2} \rho+1+2 q \rho+q^{2} \rho^{2}+q^{3} \rho\right)(-1+a) x^{2} \\
& -q(-1+a)\left(q^{3} \rho \nu a+a q^{2} \nu+a q^{3}+q \rho \nu a+q \nu a+q^{2} \rho \nu a-a q+\nu a-q \nu\right. \\
& \left.-q \rho \nu-\nu-q^{2} \rho \nu-\nu q^{2}-q^{3} \rho \nu\right) x-(1+q)(-1+a)\left(a^{2}-a-a q+1\right) ;
\end{aligned}
$$

$I_{4}(r, n, q, x)=\left(q^{3} x-1\right) a\left(q^{2} x \rho \nu+q x \nu-q+a q-1+a\right)$.

The operator $\mathbb{F}_{n}^{(r)}$ factorizes as

$$
X_{n} \mathbb{F}_{n}^{(r)}=\mathbb{S}_{n}^{(r)} \mathbb{T}_{n}^{(r)}
$$

with

$$
\begin{aligned}
X_{n}= & (q x-1)^{2}\left(q^{2} x-1\right)^{2}(x \nu-1+a) \\
& \times\left(a^{2}-a+a x \nu+1-x \nu+q x \nu a-\nu q x+\nu^{2} q x^{2}-a q x\right) P_{r-1}^{3}(q x)-a\left(q^{2} x-1\right)^{2} \\
& \times(q x-1)^{2}\left(2 \nu^{2} q x^{2}+2 q x \nu a-a q x-2 \nu q x-2 x \nu+2 a x \nu+2+2 a^{2}-3 a\right) \\
\times & P_{r-1}(x) P_{r-1}^{2}(q x)+(q x-1)^{2}\left(q^{2} x-1\right)^{2} a^{2}(a-1+\nu q x) P_{r-1}^{2}(x) P_{r-1}(q x) ; \\
\mathbb{T}_{n}^{(r)}= & a(q x-1) P_{r-1}(q x) P_{r-1}(x) \mathcal{G}_{q}^{2}-(a-1+q x \rho \nu) P_{r-1}(x) \\
& \times\left(-P_{r-1}(x) a+a P_{r-1}(q x)-P_{r-1}(q x)+P_{r-1}(q x) x \nu\right) \mathcal{G}_{q}+(x-1) P_{r-1}(q x) \\
& \times\left(-P_{r-1}(x) a+a P_{r-1}(q x)-P_{r-1}(q x)+P_{r-1}(q x) x \nu\right) .
\end{aligned}
$$


The left operator factor $\mathbb{S}_{n}^{(r)}$ is given by

$$
\mathbb{S}_{n}^{(r)}=J_{2}(r, n, q, x) \mathcal{G}_{q}^{2}+J_{1}(r, n, q, x) \mathcal{G}_{q}+J_{0}(r, n, q, x)
$$

where

$$
\begin{aligned}
J_{2}(r, n, q, x)= & P_{r-1}(q x)\left(q^{2} x-1\right)(q x-1)^{2}\left(q^{2} x \rho \nu+q x \nu-q+a q-1+a\right) \\
J_{1}(r, n, q, x)= & -(q x-1)\left(-2 a^{2} q^{2}-q x a+2 a q^{2}+a-q-3 a^{2} q+a^{3} q^{2}-x a^{2} q^{3}-q^{2}\right. \\
& +a q^{3} x+a^{3} q+a^{2} q^{3} x \rho \nu+\nu x q^{4} a^{2} \rho-a^{2}+3 a q+a^{2} q x-2 q x \nu a-2 a q^{2} x \nu \\
& +a^{2} q x \nu+q^{3} x^{2} \nu^{2} a+a^{2} q^{2} x \nu+\nu x a^{2} q^{3}+q x \nu+\nu x q^{3}-q^{3} x^{2} \nu^{2}+\nu x q^{2} \\
& -2 \nu a q^{3} x+q^{3} x \rho \nu+\nu q^{4} x \rho+q^{2} x \rho \nu-2 q^{4} \nu^{2} x^{2} \rho-q^{5} x^{2} \rho^{2} \nu^{2}+q^{5} x^{3} \rho^{2} \nu^{3} \\
& -\nu^{2} x^{2} q^{3} \rho+q^{4} x^{3} \nu^{3} \rho-q^{2} x^{2} \rho \nu^{2}+q^{5} x^{2} \rho^{2} \nu^{2} a-2 q^{2} x \rho \nu a-2 \nu x q^{4} a \rho \\
& \left.+q^{2} x \rho a^{2}-2 q^{3} x \rho \nu a+2 \nu^{2} x^{2} q^{4} a \rho+a q^{3} x^{2} \rho \nu^{2}+q^{2} x^{2} \rho \nu^{2} a\right) P_{r-1}(q x) \\
& +(q x-1) a q\left(-1+a-q+a q+\nu x q^{2}+q^{3} x \rho \nu\right)(a-1+q x \rho \nu) P_{r-1}(x) ;
\end{aligned}
$$

and

$$
\begin{aligned}
J_{0}(r, n, q, x)= & \left(-1+a-q+a q+\nu x q^{2}+q^{3} x \rho \nu\right) \\
& \times a q\left(-q x a-a+a^{2}+a x \nu+1-x \nu+q x \nu a-q x \nu+q x^{2} \nu^{2}\right) P_{r-1}(q x) \\
& -\left(-1+a-q+a q+\nu x q^{2}+q^{3} x \rho \nu\right) a^{2} q(a-1+q x \nu) P_{r-1}(x) .
\end{aligned}
$$

\section{The Stieltjes-Wigert Case}

The Stieltjes-Wigert orthogonal polynomials are defined by

$$
S_{n}(x ; q)=\frac{1}{(q ; q)_{n 1}} \Phi_{1}\left(\begin{array}{c}
q^{-n} \\
0
\end{array} \mid q ;-q^{n+1} x\right), x=q^{s},
$$

and are related to the monic Stieltjes-Wigert orthogonal polynomials $\left(P_{n}\right)_{n}$ by

$$
S_{n}(x ; q)=\frac{(-1)^{n} q^{n^{2}}}{(q ; q)_{n}} P_{n}(x) .
$$

$\left(P_{n}\right)_{n}$ satisfy the three-term recurrence equation

$$
x P_{n}(x)=P_{n+1}(x)+q^{-1-2 n}\left[1+q-q^{n+1}\right] P_{n}(x)+q^{1-4 n}\left(1-q^{n}\right) P_{n-1}(x),
$$

and the second-order $q$-difference equation

$$
q x y\left(q^{2} x\right)+\left[q x\left(1-q^{n}\right)-(q x+1)\right] y(q x)+y(x)=0,
$$

the latter equation being equivalent to Eq. (4) with

$$
\phi(x)=x, \quad \psi(x)=\frac{q x-1}{q-1}, \quad \lambda_{n}=\frac{\left(1-q^{n}\right)}{(1-q)^{2}} .
$$


The Stieltjes-Wigert weight defined by

$$
w(x)=\frac{1}{(-x ; q)_{\infty}\left(-q x^{-1} ; q\right)_{\infty}},
$$

with

$$
(a ; q)_{\infty}=\prod_{k=0}^{\infty}\left(1-a q^{k}\right)
$$

satisfies the $q$-Pearson Eq. (5) with the polynomials $\phi$ and $\psi$ given by Eq. (34).

The fourth-order $q$-difference operator $\mathbb{F}_{n}^{(r)}$ for the $r$ th associated Stieltjes-Wigert orthogonal polynomials is given by

$$
\mathbb{F}_{n}^{(r)}=I_{4}(r, n, q, x) \mathcal{G}_{q}^{4}+I_{3}(r, n, q, x) \mathcal{G}_{q}^{3}+I_{2}(r, n, q, x) \mathcal{G}_{q}^{2}+I_{1}(r, n, q, x) \mathcal{G}_{q}+I_{0}(r, n, q, x),
$$

with the notations: $\nu=q^{r}, \rho=q^{n}$ and

$$
\begin{aligned}
I_{0}(r, n, q, x)= & q x\left(q^{3} x \rho \nu+x \nu q^{2}+q+1\right) \\
I_{1}(r, n, q, x)= & -q^{4} \nu^{3} \rho(q \rho+1) x^{3}-q^{2} \nu^{2}\left(q+q^{3} \rho^{2}+2 q^{2} \rho+\rho+q \rho\right) x^{2} \\
& -q\left(\nu q^{2}+\nu-q^{2}+q \rho \nu+q^{3} \rho \nu+1+q \nu+q^{2} \rho \nu\right) x-q(q+1) ; \\
I_{2}(r, n, q, x)= & \nu^{3} q^{4}(q \rho+1)\left(q^{2} \rho^{2}+1\right) x^{3}+\nu q^{2}(q+1) \\
& \times\left(\nu q^{3} \rho^{2}-q^{2} \rho+\nu q^{2} \rho^{2}+q^{2} \rho \nu+q \rho \nu+q \nu-q+\nu\right) x^{2} \\
& +2 q\left(-q^{2}-q+q^{2} \rho \nu+\nu q^{2}+q^{3} \rho \nu+q \rho \nu+q \nu+\nu\right) x+(q+1)^{2} ; \\
I_{3}(r, n, q, x)= & -q^{6} \nu^{3} \rho(q \rho+1) x^{3}-q^{3} \nu^{2}\left(q^{2} \rho+2 q \rho+q^{2} \rho^{2}+q^{3} \rho+1\right) x^{2} \\
& -q\left(q \nu-q+q^{2} \rho \nu+\nu q^{2}+q^{3} \rho \nu+q \rho \nu+\nu+q^{3}\right) x-q-1 ; \\
I_{4}(r, n, q, x)= & q^{3} x\left(q^{2} x \rho \nu+q+q x \nu+1\right) .
\end{aligned}
$$

The operator $\mathbb{F}_{n}^{(r)}$ factorizes as

$$
X_{n} \mathbb{F}_{n}^{(r)}=\mathbb{S}_{n}^{(r)} \mathbb{T}_{n}^{(r)}
$$

with

$$
\begin{aligned}
X_{n}= & q^{5} x^{4}(1+x \nu)\left(1+x \nu-q x+\nu q x+\nu^{2} q x^{2}\right) P_{r-1}(q x)^{3} \\
- & q^{5} x^{4}\left(2 \nu^{2} q x^{2}+2 \nu q x+2 x \nu-q x+2\right) P_{r-1}(x) P_{r-1}(q x)^{2} \\
+ & q^{5} x^{4}(1+\nu q x) P_{r-1}(x)^{2} P_{r-1}(q x) \\
\mathbb{T}_{n}^{(r)}= & q x P_{r-1}(x) P_{r-1}(q x) \mathcal{G}_{q}^{2}-P_{r-1}(x)\left(-P_{r-1}(x)\right. \\
& \left.\quad+P_{r-1}(q x)+P_{r-1}(q x) x q^{r}\right)\left(1+q x q^{n} q^{r}\right) \mathcal{G}_{q} \\
& +x P_{r-1}(q x)\left(-P_{r-1}(x)+P_{r-1}(q x)+P_{r-1}(q x) x q^{r}\right) .
\end{aligned}
$$


The left operator factor $\mathbb{S}_{n}^{(r)}$ is given by

$$
\mathbb{S}_{n}^{(r)}=J_{2}(r, n, q, x) \mathcal{G}_{q}^{2}+J_{1}(r, n, q, x) \mathcal{G}_{q}+J_{0}(r, n, q, x),
$$

where

$$
\begin{aligned}
& J_{2}(r, n, q, x)=q^{3} x^{3} P_{r-1}(q x)\left(q^{2} x \rho \nu+q+q x \nu+1\right) \\
& J_{1}(r, n, q, x)=q x\left(q^{3} x \rho \nu+x \nu q^{2}+q+1\right)(1+q x \rho \nu) P_{r-1}(x) \\
& \quad-q x\left(x \nu q^{2}+q x \rho \nu+x \nu+q-q^{2} x+q^{2} x^{2} \nu^{2}+\nu^{3} q^{3} x^{3} \rho+q^{3} x \rho \nu\right. \\
& \quad+x+\nu^{3} x^{3} \rho^{2} q^{4}+2 \nu^{2} q^{3} x^{2} \rho+\nu^{2} x^{2} q \rho+\nu^{2} q^{2} x^{2} \rho+\nu^{2} q^{4} x^{2} \rho^{2} \\
& \left.\quad+1+q x \nu+q^{2} x \rho \nu\right) P_{r-1}(q x) ;
\end{aligned}
$$

and

$$
\begin{aligned}
J_{0}(r, n, q, x)= & -(q x \nu+1)\left(q^{3} x \rho \nu+x \nu q^{2}+q+1\right) P_{r-1}(x) \\
& +\left(-q x+q x \nu+1+x \nu+q x^{2} \nu^{2}\right)\left(q^{3} x \rho \nu+x \nu q^{2}+q+1\right) P_{r-1}(q x) .
\end{aligned}
$$

\section{Extension of Results}

1. Let $\nu$ be a real number with $\nu \geq 0$ and $\left(P_{n}^{(r)}\right)_{n}$ the family of polynomials defined by

$$
P_{n+1}^{(\nu)}(x)=\left(x-\beta_{n+\nu}\right) P_{n}^{(\nu)}(x)-\gamma_{n+\nu} P_{n-1}^{(\nu)}(x), n \geq 1,
$$

with the initial conditions

$$
P_{0}^{(\nu)}(x)=1, P_{1}^{(\nu)}(x)=x-\beta_{\nu},
$$

where $\beta_{n+\nu}$ and $\gamma_{n+\nu}$ are the coefficients $\beta_{n}$ and $\gamma_{n}$ of Eq. (8) with $n$ replaced by $n+\nu$.

We assume that the starting family $\left(P_{n}\right)_{n}$ defined in Eq. (8) is $q$-classical. The coefficients $\beta_{n}$ and $\gamma_{n}$ are therefore rational functions in the variable $q^{n}[19,23]$ and the coefficients $\beta_{n+\nu}$ and $\gamma_{n+\nu}$ are well-defined. When $\gamma_{n+\nu} \neq 0, \forall n \geq 1$, the family $\left(P_{n}^{(r)}\right)_{n}$, thanks to Favard's theorem [6,8], is orthogonal and represents the associated of the family $\left(P_{n}\right)_{n}$ with real order of association (see for example, Ref. [5]). Theorems 2 and 5, can be generalized to the real order of association $\nu$ (with $\nu \geq 0$ ). The proof is similar to those given in Ref. [11,12] for the continuous and discrete cases.

2. The factorization pointed out in Eq. (26) (see also Eq. (29)) can be used to prove the following proposition, whose proof is similar to those given in Refs. [11,12] for orthogonal polynomials of continuous and discrete variables.

Proposition 1 Two linearly independent solutions of the q-difference equation

$$
\mathbb{S}_{n}^{(r)}(y)=0,
$$

are

$$
E_{n}^{(r)}(x)=\mathbb{T}_{n}^{(r)}\left(C_{n}^{(r)}(x)\right), \quad F_{n}^{(r)}(x)=\mathbb{T}_{n}^{(r)}\left(D_{n}^{(r)}(x)\right),
$$


where the operators $\mathbb{S}_{n}^{(r)}$ and $\mathbb{T}_{n}^{(r)}$ are those defined in $E q$. (26) and the functions $C_{n}^{(r)}(x)$ and $D_{n}^{(r)}(x)$ given by Eq. (29).

The previous proposition can also be generalized to real order of association (see Refs. $[11,12]$ for orthogonal polynomials of continuous and discrete variables cases).

Remark 1 Using the previous proposition one can solve a family of second-order $q$-difference equations with polynomial coefficients. This proposition, given for the associated $q$-classical orthogonal polynomials can be used to solve the $q$-difference equation $\mathbb{S}_{n}(y)=0$ where $\mathbb{S}_{n}$ is the left factor of the factored form of the fourth-order $q$ difference operator $\mathbb{F}_{n}\left(X_{n} \mathbb{F}_{n}=\mathbb{S}_{n} \mathbb{T}_{n}\right)$ for other modifications of $q$-classical orthogonal polynomials (see Section 2).

Theorem 1, valid for orthogonal polynomials $\left(\bar{P}_{n}\right)_{n}$ satisfying Eq. (10), with the initial family $\left(P_{n}\right)_{n}$ being $q$-classical can be extended as in Refs. [11,12] to more general situations.

\section{Acknowledgements}

We are very grateful to the Alexander von Humboldt Foundation whose grant (Fellowship No IV KAM 1070235) and the equipment donation (No V-8151/03137) accorded to the first author Mama Foupouagnigni were decisive for the realization of this work. We would also like to thank the anonymous referees for their careful reading of the manuscript and their helpful suggestions.

\section{References}

[1] R. Álvarez-Nodarse and J. Arvestú, On the $q$-polynomials in the exponential lattice $x(s)=c_{1} q^{s}+c_{3}$, Integral Transform and Special Functions, 8 (1999), 299-324.

[2] R. Álvarez-Nodarse and J.C. Medem, $q$-Classical polynomials and $q$-Askey and Nikiforov-Uvarov Tableaux, J. Comp. Appl. Math., 135 (2001), 157-196.

[3] N.M. Atakishiyev, M. Rahman and S.K. Suslov, On classical orthogonal polynomials, Constr. Approx., 11 (1995), 181-226.

[4] S. Belmehdi and A. Ronveaux, Polynômes associés des polynômes orthogonaux classiques. Construction via REDUCE, In: L. Arias, et al., eds, Orthogonal Polynomials and their Applications Universidad de Oviedo, 1989, pp 72-83.

[5] J. Bustoz and M.E.H. Ismail, The associated ultraspherical polynomials and their $q$-analogues, Can. J. Math., 34 (1982), 718-736.

[6] T.S. Chihara, Introduction to Orthogonal Polynomials, Gordon and Breach, New York, 1978.

[7] J. Dini, Sur les formes linéaires et polynômes orthogonaux de Laguerre-Hahn Thèse de Doctorat, Université Pierre et Marie Curie, Paris VI, 1988.

[8] J. Favard, Sur les polynômes de Tchebicheff, C. R. Acad. Sci. Paris, 200 (1935), 2052-2053.

[9] M. Foupouagnigni, Laguerre-Hahn Orthogonal Polynomials with respect to the Hahn Operator, Fourth-order Difference Equation for the rth Associated and the Laguerre-Freud Equations for the Recurrence Coefficients, Ph.D. Thesis, Université Nationale du Bénin, Bénin, 1998.

[10] M. Foupouagnigni, A. Ronveaux and W. Koepf, The fourth-order $q$-difference equation satisfied by the first associated $q$-classical orthogonal polynomials, J. Comput. Appl. Math., 101 (1999), 231-236.

[11] M. Foupouagnigni, W. Koepf and A. Ronveaux, Factorization of fourth-order differential equations for perturbed classical orthogonal polynomial, J. Comput. Appl. Math., 162 (2004), 299-326.

[12] M. Foupouagnigni, W. Koepf and A. Ronveaux, On fourth-order difference equations for orthogonal polynomials of a discrete variable: derivation, factorization and solutions, J. Diff. Eqn. Appl., 9 (2003), $777-804$.

[13] M. Foupouagnigni, A. Ronveaux and M.N. Hounkonnou, The fourth-order $q$-difference equation satisfied by the associated orthogonal polynomials of the $D_{q}$-Laguerre-Hahn class, J. Diff. Eqn. Appl., 7 (2001), 445-472.

[14] M. Foupouagnigni and A. Ronveaux, The fourth-order difference equation satisfied by the co-recursive of the $q$-classical orthogonal polynomials, J. Comput. Appl. Math., 133 (2001), 355-365.

[15] M. Foupouagnigni and A. Ronveaux, The fourth-order difference equation satisfied by the co-recursive associated orthogonal polynomials of the $D_{q}$ Laguerre-Hahn class, J. Comput. Appl. Math., 153 (2002), $213-223$. 
[16] W. Hahn, Über Ortogonalpolynome die q-Differenzengleichungen genügen, Math. Nachr, 2 (1949), 4-34.

[17] M.E.H. Ismail, Difference equations and quantized discriminants for $q$-orthogonal polynomials, Adv. Appl. Math., 30 (2003), 562-589.

[18] M.E.H. Ismail and P. Simeonov, A fourth-order $q$-difference equation for the associated discrete $q$-orthogonal polynomials, J. Math., 32 (2002), 679-690.

[19] W. Koepf and D. Schmersau, Recurrence equations and their classical orthogonal polynomial solutions, Appl. Math. Comput., 128 (2002), 303-327.

[20] R. Koekoek and R. Swarttouw, The Askey-scheme of hypergeometric orthogonal polynomials and its $q$ analogue, Report no. 98-17, Faculty of Information Technology and Systems, Delft University of Technology, 1998.

[21] F. Marcellán, J.S. Dehesa and A. Ronveaux, On orthogonal polynomials with perturbed recurrence relations, J. Comp. Appl. Math., 30 (1990), 203-212.

[22] J.C. Medem, Poliomios ortogonales $q$-semiclásicos, Ph.D. Dissertation, Universidad Politécnica de Madrid, 1996.

[23] J.C. Medem, R. Álvarez-Nodarse and F. Marcellán, On the $q$-polynomials: a distributional study, J. Comp. Appl. Math., 135 (2001), 197-233.

[24] M.B. Monagan, K.O. Geddes, K.M. Heal, G. Labahn, S.M. Vorkoetter, J. McCarron and P. DeMarco, Maple 8, Waterloo Maple, Inc.

[25] A.F. Nikiforov and V.B. Uvarov, Special Functions of Mathematical Physics Birkhäuser, Basel, Boston, 1988

[26] A.F. Nikiforov, S.K. Suslov and V.B. Uvarov, Classical Orthogonal Polynomials of a Discrete Variable, Springer, Berlin, 1991.

[27] A. Ronveaux and W. Van Assche, Upward extension of the jacobi matrix for orthogonal polynomials, J. Approx. Theory, 86 (1996), 335-357.

[28] S.K. Suslov, The theory of difference analogues of special functions of hypergeometric type, Russ. Math. Surveys, 44 (1989), 227-278. 